\title{
A note on quantum Bohlin transformation
}

\author{
A. Nersessian用, V. Ter-Antonyan叫, M.Tsulaiaf \\ Bogolyubov Laboratory of Theoretical Physics, \\ Joint Institute for Nuclear Research \\ Dubna, Moscow region, 141980, Russia
}

\begin{abstract}
It is shown, that the reduction of the circular quantum oscillator by the $Z_{2}$ group action results to the two systems: a two-dimensional hydrogen atom, and a "charge - charged magnetic vortex" one, with the spin $\frac{1}{2}$. Analogously, the $Z_{N}$ reduction of the two-dimensional system with the central potential $r^{2(N-1)}$ results into $N$ bound "charge - magnetic vertex" systems with the interaction potential $r^{2(1 / N-1)}$ and spins $\sigma=\frac{k}{N}, k=0,1, \ldots,(N-1)$.
\end{abstract}

PACS number : 03.65.Ge

*e-mail:nerses@thsun1.jinr.dubna.su

$\dagger$ †-mail:terant@thsun1.jinr.dubna.su

‡e-mail:tsulaia@thsun1.jinr.dubna.su 
1. Introduction. As it was established by Bohlin [四], trajectories of the circular oscillator and the two-dimensional Kepler problem are connected by the transformation

$$
w=z^{2}
$$

where the complex coordinates $z$ and $w$ parametrize the position of a particle in the oscillator and Kepler problem.

Due to (四), to the tracing along the ellipse in the oscillator problem there corresponds the double tracing of the ellipse in the Kepler problem. In the quantum case, this is reflected in the fact, that the circular oscillator is transferred by (1) into an (non-physical) Coulomb problem on the two-sheet Riemann surface.

In this note we show that if prior to the transformation (11) we perform the reduction of the oscillator by the $Z_{2}$ group action, given the parity operator, the oscillator splits into two physical systems: a two-dimensional hydrogen atom (even states) and a bound "charge-charged magnetic vortex" system (odd states). The second system has the eigen angular momentum (spin) $\frac{1}{2}$ and its energy levels are shifted with respect to the levels of the first system.

We have also shown that the two-dimensional system with the potential $|z|^{2(N-1)}$ after the reduction by the $Z_{N}$ group action and subsequent transformation $w=z^{N}$ splits into $N$ "charge- magnetic vortex" systems, interacting with the potential $|w|^{-2(1-1 / N)}$, which have eigen angular momenta $\sigma=\frac{k}{N}, k=0,1, \ldots,(N-1)$.

2.Bohlin transformation. Let us consider the Schrödinger equation for the isotropic oscillator on the plane $\dot{\mathbb{C}}=\mathbb{C}-\{0\}$

$$
4 \partial_{z} \bar{\partial}_{z} \Psi+\frac{2 \mu}{\hbar^{2}}\left(E-\frac{\mu \omega^{2}}{2} z \bar{z}\right) \Psi=0,
$$

where $\partial_{z}=\partial / \partial z, \bar{\partial}_{z}=\partial / \partial \bar{z}$.

Reduce Eq.(2) by the group $Z_{2}$, having restricted ourselves to even $(\sigma=0)$ or odd $\left(\sigma=\frac{1}{2}\right)$ solutions of Eq. (2)

$$
\Psi_{\sigma}(z, \bar{z})=\psi_{\sigma}\left(z^{2}, \bar{z}^{2}\right) \mathrm{e}^{2 i \sigma \arg z}
$$

and then perform the transformation

$$
w=\lambda z^{2}, \quad \lambda=\left(\frac{\mu \omega}{\hbar}\right)^{\frac{1}{2}}
$$

Let us call a set of these transformations the quantum Bohlin transformation (note that in contrast with formula (11) the coordinates $z$ and $w$ in formula (4) have the same dimension).

According to Eq.(3), the wave functions $\psi_{\sigma}$ satisfy the condition

$$
\psi_{\sigma}(|w|, \arg w+2 \pi)=\psi_{\sigma}(|w|, \arg w),
$$

which imply that the range of definition $\arg w \in[0,4 \pi)$ can be restricted, without loss of generality, to $\arg w \in[0,2 \pi)$.

Thus, the quantum Bohlin transformation reduces the oscillator to the system on the plane rather than on the two-sheet Riemann surface. 
One can easily see that the quantum Bohlin transformation reduces eq.(2) to the form

$$
\frac{1}{2 \mu} \hat{p}_{\sigma} \hat{p}_{\sigma}^{+} \psi(w, \bar{w})-\left(\frac{\alpha}{|w|}+\mathcal{E}\right) \psi_{\sigma}=0
$$

where

$$
\hat{p}_{\sigma}=-2 i \hbar \partial_{w}-\frac{i \hbar \sigma}{2 w}
$$

and the parameters $\mathcal{E}$ and $\alpha$ are determined by the conditions

$$
\mathcal{E}=-\frac{\mu \omega^{2}}{8 \lambda^{2}}, \quad \alpha=\frac{E}{4 \lambda}
$$

and have the meaning of the energy and coupling constant of the system obtained.

Equation (5) can be interpreted as the Schrödinger equation of a nonrelativistic particle with electric charge $e$ in the static electromagnetic field, determined by the potential

$$
\mathcal{A}=\left(\phi, A_{w}\right): \quad \phi=-\frac{Q}{|w|}, \quad A_{w}=\frac{i g}{w},
$$

where

$$
Q=\alpha, \quad \frac{e g}{\hbar c}=\sigma=0, \frac{1}{2}
$$

It is to be noted that $\phi$ is the potential of the two-dimensional Coulomb field of a particle with charge $Q$, and $A_{w}$ is a vector potential of an infinitely thin solenoid- flux tube ( magnetic vortex, in two dimensional interpretation): to it there corresponds zeroth strength of the magnetic field $B=\operatorname{rot} A_{w}=0(w \in \dot{\mathbb{C}})$ and nonzero magnetic flux $2 \pi g$.

Consequently, the potential $\mathcal{A}=\left(\phi, A_{w}\right)$ defines the electromagnetic field the twodimensional charged magnetic vortex (two-dimensional dyon) with the electric $Q$ and magnetic $g$ charges.

Thus, having reduced the two-dimensional oscillator by the $Z_{2}$ group action, we have obtained a two-dimensional hydrogen atom at $\sigma=0$ and a "charge-charged magnetic vortex" system ("charge-two-dimensional dyon") at $\sigma=\frac{1}{2}$.

The Hamiltonian of the circular oscillator has hidden symmetry so(3)

$$
\left\{I, I^{+}\right\}=-2(\hbar \omega)^{2} J, \quad\{I, J\}=-2 I, \quad\left\{I^{+}, J\right\}=2 I^{+}
$$

generated by the angular momentum operator

$$
J=2(z \partial-\bar{z} \bar{\partial})
$$

and the vector (complex) constant of motion

$$
I=-\frac{2 \hbar^{2}}{\mu} \bar{\partial}_{z}^{2}+\frac{\mu \omega^{2}}{2} z^{2} .
$$


These operators are even $\left(Z_{2}\right.$-invariant $)$ and, therefore, the reduced systems also have hidden so(3) symmetry that is generated by these operators reduced by the quantum Bohlin transformation to the form

$$
\begin{aligned}
& J \rightarrow 2 J_{\sigma}, \quad J_{\sigma}=\frac{i}{\hbar}\left(w \hat{p}_{\sigma}-\bar{w} \hat{p}_{\sigma}^{+}\right) \\
& I \rightarrow 4 \lambda I_{\sigma} \quad I_{\sigma}=\frac{i}{2 \mu}\left(J_{\sigma} \hat{p}_{\sigma}+\hat{p}_{\sigma} J_{\sigma}\right)-\frac{\alpha w}{|w|} .
\end{aligned}
$$

Note that $J_{\sigma}$ is the angular momentum operator of the reduced system and $I_{\sigma}$ is its Runge-Lenz vector.

It is seen from expression (12) that the eigenvalues of the angular momenta of the reduced system and the oscillator, $m_{\sigma}$ and $M$, are related by the expression $M=2 m_{\sigma}$, from which follows

$$
m_{\sigma}= \pm \sigma, \pm(1+\sigma), \pm(2+\sigma), \ldots
$$

Using expressions (6), we easily get from the oscillator energy spectrum

$$
E=\hbar \omega\left(2 N_{r}+|M|+1\right), N_{r}=0,1,2, \ldots,
$$

the energy spectrum of the reduced system

$$
\mathcal{E}_{\sigma}=-\frac{\mu \alpha^{2}}{2 \hbar^{2}\left(N_{r}+\left|m_{\sigma}\right|+\frac{1}{2}\right)^{2}}
$$

Hence, the "charge-charged magnetic vortex" system has the eigen angular moment $\sigma=\frac{1}{2}$ and the energy spectrum is shifted with respect to the energy spectrum of the twodimensional hydrogen atom, i. e. the Aharonov - Bohm effect takes place.

Note also that composites "charge - magnetic vortex" are anyons, particles with fractional statistics and spin equal to their eigen angular momentum (see, e.g. [2]).

Finally, using expression (3), we find from the wave functions of the oscillator

$$
\Psi_{N_{r}, M}(z, \bar{z})=C_{N_{r},|M|}(\lambda|z|)^{|M|} \mathrm{e}^{-\frac{\lambda^{2}|z|^{2}}{2}} L_{N_{r}}^{|M|}\left(\lambda^{2}|z|^{2}\right) \mathrm{e}^{i M \arg z} / \sqrt{2} \pi .
$$

the wave functions of the reduced system

$$
\psi_{\sigma, N_{r}, m_{\sigma}}=\frac{C_{N_{r}, 2\left|m_{\sigma}\right|}}{2 \sqrt{N_{r}+\left|m_{\sigma}\right|+\frac{1}{2}}}\left(\lambda_{N_{r}, m_{\sigma}}|w|\right)^{\left|m_{\sigma}\right|} \mathrm{e}^{-\frac{\lambda_{N_{r}, m_{\sigma}}|w|}{2}} L_{N_{r}}^{2\left|m_{\sigma}\right|}\left(\lambda_{N_{r}, m_{\sigma}}|w|\right) \mathrm{e}^{i\left(m_{\sigma}-\sigma\right) \arg w} / \sqrt{2} \pi .
$$

Here $L_{p}^{s}(x)$ are the generalized Laguerre polynomials, related with the confluence hypergeometric function by

$$
L_{p}^{s}(x)=\frac{\Gamma(s+p+1)}{\Gamma(s+1)} F(-p, s+1, x),
$$

and the constants $C_{N_{r},|M|}$ and $\lambda_{N_{r}, m_{\sigma}}$ have the form

$$
C_{N_{r},|M|}=\frac{\sqrt{2} \lambda}{\sqrt{N_{r} !\left(N_{r}+|M|\right) !}}, \quad \lambda_{N_{r}, m_{\sigma}}=\frac{2}{r_{B}\left(N_{r}+m_{\sigma}+\frac{1}{2}\right)} .
$$


where $r_{B}=\frac{\hbar^{2}}{\mu \alpha}$ is the "Bohr radius" of a hydrogen atom.

3. Generalization. In a popular book by Arnold [3] the generalization of the Bohlin transformation

$$
w=z^{N}
$$

is given, connecting the classical trajectories of the two-dimensional systems with the potentials $|z|^{2 a}$ and $|w|^{2 b}$, where

$$
(a+1)(b+1)=1, \quad N=a+1 .
$$

What does the transformation (19) lead to in the quantum case?

We restrict ourselves, for simplicity, to the case, when the parameter $N$ is a natural number.

Let us consider on the complex plane $\dot{\mathbb{C}}$ the spectral problem (here and hereafter $\hbar=\mu=c=1)$

$$
\left\{\begin{array}{c}
\left(-2 \partial_{z} \bar{\partial}_{z}+A|z|^{2(N-1)}-E\right) \Psi=0 \\
2\left(z \partial_{z}-\bar{z} \bar{\partial}_{z}\right) \Psi=M \Psi
\end{array},\right.
$$

with the single-valued wave functions

$$
\Psi(z, \bar{z})=R_{M, E}(|z|) \mathrm{e}^{i M \arg z} \quad M=0, \pm 1, \pm 2, \ldots
$$

The transformation (19) maps the system 21 into the one on the $N$-sheet Riemann surface. Therefore, reduce the system (21) by the group $Z_{N}$, substituting the wave functions of the form

$$
\Psi_{\sigma}(z, \bar{z})=\psi_{\sigma}\left(z^{N}, \bar{z}^{N}\right) \mathrm{e}^{N i \sigma \arg z}, \quad \sigma=\frac{k}{N}, \quad k=0,1, \ldots(N-1)
$$

into the system of equations (21) and then performed the transformation (19), we get

$$
\left\{\begin{array}{c}
\left(\frac{1}{2} p_{\sigma} \bar{p}_{\sigma}+\alpha|w|^{2\left(\frac{1}{N}-1\right)}-\mathcal{E}\right) \psi(w, \bar{w})=0 \\
2 i\left(w p_{\sigma}-\bar{w} \bar{p}_{\sigma}\right) \psi=m_{\sigma} \psi
\end{array}\right.
$$

where

$$
p_{\sigma}=-2 i \partial_{w}-\frac{i \sigma}{w}, \quad \mathcal{E}=-\frac{A}{N^{2}}, \quad \alpha=-\frac{E}{N^{2}},
$$

and the eigenvalues of the angular momentum of the system are

$$
m_{\sigma}= \pm \sigma, \pm(1+\sigma), \pm(2+\sigma), \ldots
$$

Thus, as a result of the reduction by the group $Z_{N}$ the system (21) disintegrated into $N$ bound "charge-magnetic vortex" systems with the interaction potential $\beta|w|^{-2\left(\frac{1}{N}-1\right)}$ and eigen angular momenta $\sigma=0,1 / N, 2 / N, \ldots,(N-1) / N$.

4. Conclusion We have shown that the quantum Bohlin transformation splits the two-dimensional oscillator into two systems having the Coulomb symmetry: the twodimensional hydrogen atom and the "charge-charged magnetic vortex" system with a 
half-integer angular momentum. However, this is not the only connection between the oscillator and topologically nontrivial quantum systems with hidden Coulomb symmetry. Thus, the reduction of the four-dimensional oscillator by the group $U(1)$ together with the Kuustanheimo-Steifel [5] transformation results in the three-dimensional bound system "charge- $U(1)$-dyon" [4] that is described by the Hamiltonian

$$
\hat{H}=\frac{\hbar^{2}}{2 \mu} \hat{\pi}^{2}+\frac{\hbar^{2} s^{2}}{2 \mu r^{2}}-\frac{\alpha}{r}, \quad s=0, \pm 1 / 2, \pm 1, \ldots,
$$

where

$$
\left[\hat{\pi}_{i}, \hat{\pi}_{j}\right]=i s \epsilon_{i j k} \frac{x_{k}}{r^{3}}, \quad\left[\hat{\pi}_{i}, x_{j}\right]=-i \delta_{i j}
$$

and $\mathbf{r} / r^{3}$ is the magnetic field strength of the $U(1)$-monopole with the unit magnetic charge. Note that the Hamiltonian of the system (5) can be represented in an analogous form

$$
\hat{H}_{\sigma}=-\frac{4 \hbar^{2}}{2 \mu} \partial_{w} \bar{\partial}_{w}+\frac{\hbar^{2} \sigma^{2}}{2 \mu|w|^{2}}-\frac{\alpha}{|w|} .
$$

Acknowledgement. We are grateful to V. Osipov for useful discussions.

\section{References}

[1] K. Bohlin, Bull. Astr., 28(1911), 144

[2] F. Wilczek, Phys. Rev. Lett., 48 (1982),114; in Fractional Statistics and Anyon Superconductivity, edited by F. Wilczek (World Scientific, Singapoore 1990);

[3] V.I.Arnold, Huygens and Barrow, Newton and Hooke, "Nauka" Publ., Moscow, 1989 (in Russian, there is English translation)

[4] D. Zwanziger, Phys. Rev., 176, (1968) 1480

[5] V.Ter-Antonyan, A.Nersessian, Mod. Phys. Lett. A10(1995) 2633; 